\title{
Inside the black box
}

\section{The review process is at the heart of scientific publishing. We would like to share with our readers some of the considerations that go into finding the best possible set of referees for each paper.}

Much is at stake for authors during peer review at Nature Geoscience. But in order to ensure a rigorous evaluation of our papers, it is essential that authors have no control over the choice of referees (except for the option to exclude one or two individuals that they have good reason to believe would not judge their paper objectively). In this high-stake low-control situation, it is essential that authors trust us - the editors - to choose knowledgeable, fair and constructive referees.

It is not in our interest to decline publication of good papers: the journal thrives on bringing to light new ideas and insights that move the geosciences along. Instead, the purpose of the peer review evaluation is to make clear which conclusions of each article are firmly demonstrated - and what else the authors may be able to tease out of their data with a little more work. We can then decide whether we feel a paper will be of interest to our readers. For that, we need a panel of referees whose combined expertise covers all aspects of a paper, without personal stakes in the subject matter at hand.

Ideally, we like diversity among referees. We continuously bring in new reviewers to ensure that we reflect the make-up of the scientific community: no individual scientist has reviewed more than 12 papers for us in the almost nine years since we opened for submissions. We often assign an early career researcher, who will have time to look at the details, along with a more senior figure, who can assess the overview and implications; we check for collaborations with the authors and exclude potential referees who have recently published with the authors. For each panel of referees, we try to find people with a good

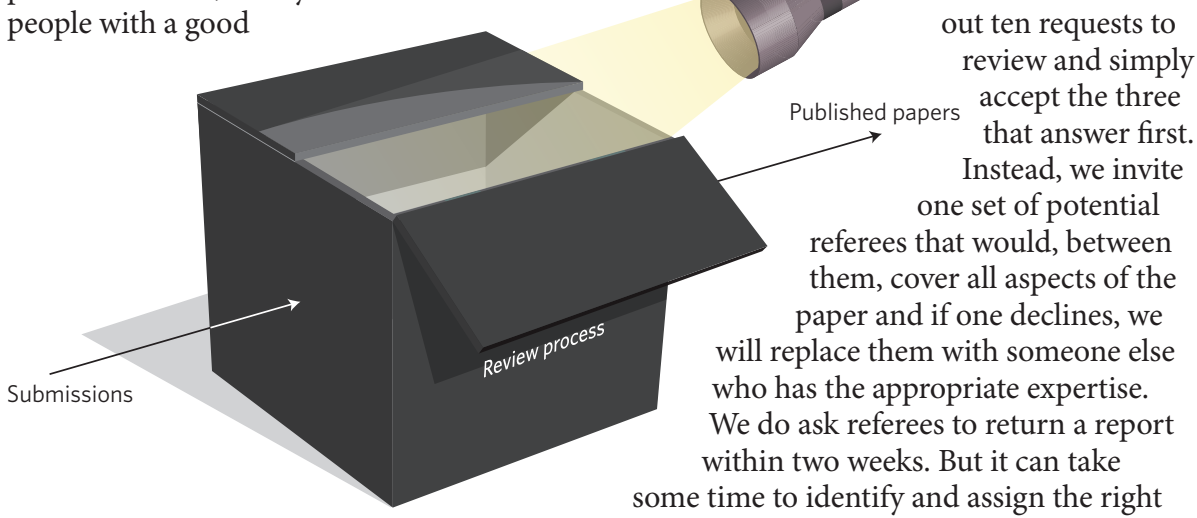

geographical spread, and avoid assigning referees who have worked closely together, to obtain a representative range of opinion.

We do our best to avoid personal or scientific conflicts - we usually rule out people at the same institution as the authors as well as scientists we know to have taken strong sides (either way) on the issue discussed in the paper. One of the insights we gain from attending meetings and visiting institutions is an understanding of this more informal fabric of the communities we serve.

Nevertheless, no processes can be perfect, and we must rely on the fairness of the referees we ask to assess papers to disclose any conflicts of interest that we are not aware of. Such conflicts can include collaborations too recent to appear in the published record or personal relationships with one of the authors (we have had people decline to review because an author was about to start a postdoc position with them, or because their ex-boyfriend was on the author list). A good reason to decline would also be an uncomfortably close focus of their own research - whether a potential referee is about to publish the same findings or the exact opposite, it would be difficult for them to be objective. We encourage referees to let us know how they have interacted with the authors in the past, or their topic of research, if in doubt - we can then make a judgement call whether it would be appropriate for them to act as referee.

To ensure the right balance of knowledge on our referee panels (while avoiding the use of more referees than necessary), we

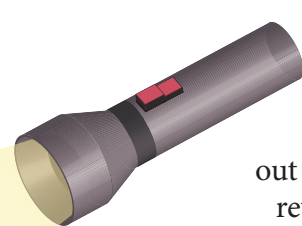
never take a scatter-gun approach: we do not send ten requests to view and simply accept the three that answer first. Instead, we invite ne set of potential referees that would, between them, cover all aspects of the paper and if one declines, we will replace them with someone else who has the appropriate expertise. We do ask referees to return a report ome time to identify and assign the right referees (and occasionally, referees drop out at a late stage and we need to start all over again), so it takes somewhat longer to make a decision. We aim for about 6 to 7 weeks from submission to a first decision after review.

We value consistency in the review process and avoid, where possible, changes or additions of referees in follow-up rounds of review. However, if reviewers who had significant concerns are unable to provide an assessment of a revised manuscript and their expertise is not represented within the remaining panel, we will need to assign a replacement referee.

Occasionally, a deadlock arises between one of our referees and the authors that cannot be resolved through further arguments. If we feel that the decision to publish or not hinges on only one referee's technical comments, and the dispute cannot be settled through revision and response, we can consider bringing in a fresh referee for adjudication. For those cases, we distil the point of contention into a concise and specific question. We then ask the authors to lay out their case, detail their responses to the negative referee and provide a sufficient number of suggestions of adjudicators to grant anonymity, usually at least half a dozen. We suggest that they choose people they trust to be fair, independent and knowledgeable about the issue at hand. We apply particularly careful checks to these suggested adjudicators, and prefer referees that we have worked with before and found to be excellent. If none of the suggestions work from our point of view, we identify an adjudicator that we trust. Their assessment will then be crucial for our ultimate decision.

We rely on our referees' knowledge and willingness to help. Without them, we could not make an informed decision of whether we feel that a paper advances the geosciences sufficiently to offer the broad appeal we are looking for. The vast majority of the scientists that we ask to review for us spend a lot of time and thought on their reports, and the papers are improved as a result of that critical review process.

We would like to take this opportunity to extend our sincere thanks to the 8,750 excellent scientists who have reviewed our papers since we opened for submissions in April 2007. 